\title{
Factors influencing use of a performance measurement system in a rehabilitation hospital
}

\author{
Diana Zidarov*1,2,3, Claude Sicotte ${ }^{4}$, Anita Menon ${ }^{1}$, Marie-Christine Hallé ${ }^{3,5}$, Lise Poissant ${ }^{2,3,6}$ \\ ${ }^{1}$ School of Physical and Occupational Therapy, McGill University, Montreal, Quebec, Canada \\ ${ }^{2}$ Institute of Rehabilitation Gingras-Lindsay of Montreal, Montreal, Quebec, Canada \\ ${ }^{3}$ Centre for Interdisciplinary Research in Rehabilitation, Montreal, Quebec, Canada \\ ${ }^{4}$ Health Administration Department, School of Public Health, University of Montreal, Quebec, Canada \\ ${ }^{5}$ Centre for Medical Education, Faculty of Medicine, McGill University, Montreal, Quebec, Canada \\ ${ }^{6}$ School of Rehabilitation, University of Montreal, Montreal, Quebec, Canada
}

Received: June 16, 2016

DOI: $10.5430 /$ jha.v5n5p79
Accepted: August 3, 2016

Online Published: August 9, 2016

URL: http://dx.doi.org/10.5430/jha.v5n5p79

\begin{abstract}
Background: Rehabilitation hospitals, like other healthcare organizations, are under increasing pressure to apply management tools such as performance measurement systems (PMSs). PMS implementation represents a major challenge as it involves significant organizational change. This study explored how a PMS was used in a rehabilitation hospital and what were the factors explaining its use.

Methods: A qualitative longitudinal study was conducted. Two data sources were used: interviews with hospital directors and organizational documents. Semi-structured interviews were conducted pre-implementation $(n=7)$ and 10 months postimplementation $(n=7)$ of the PMS. A total of 111 documents produced between 2011 and 2014 were reviewed. The Consolidated Framework for Implementation Research (CFIR) was used as a conceptual framework for data collection and analysis.

Results: Decision makers used the PMS mostly for monitoring and accountability purposes and also for promoting their organization's performance and enhancing the organization's credibility. It was rarely used to trigger change management projects. Major barriers to PMS use were the lack of planning of the implementation process, available resources and the perceived quality of the developed PMS. Major facilitators for PMS implementation were related to continuous leadership engagement, specific PMS characteristics (perceived advantages, lack of complexity), quality of communications, and a perceived need for a PMS. Some key recommendations are proposed to decision makers that may enhance PMS use.

Conclusions: PMS use is influenced by multiple factors, however the positive or negative influence of each factor is context dependent. Key recommendations are proposed to decision makers that may enhance PMS use.
\end{abstract}

Key Words: Performance measurement system, Rehabilitation, Qualitative study, Implementation, Healthcare decision makers

\section{INTRODUCTION}

Assessing performance of public services has become a central issue in public administration, ${ }^{[1]}$ and rehabilitation hospitals are indeed no exception. ${ }^{[2-4]}$ Rehabilitation hospitals provide transitional care between acute care hospitals and home-based services and this creates administrative and managerial challenges that are unique to these organizations. For example, basic processes such as admission and discharge of clients take a different perspective for a rehabilitation hospital as these processes will be largely influenced by the

\footnotetext{
*Correspondence: Diana Zidarov; Email: diana.zidarov@mcgill.ca; Address: School of Physical and Occupational Therapy, McGill University; Institute of Rehabilitation Gingras-Lindsay of Montreal; Centre for Interdisciplinary Research in Rehabilitation, Montreal, Quebec, Canada.
} 
previous or next health care provider involved. The transitional care provided by rehabilitation hospitals and their interdependence on other organizations within the continuum of care, adds to the complexity of assessing performance of these settings. It is through a comprehensive, integrated and systematic assessment of the organization's performance, using management tools such as performance measurement systems (PMSs), that high quality services could be provided. Nevertheless, PMS implementation represents a major challenge for organizations as it involves significant organizational change. ${ }^{[5]}$ Although several studies have focused on the development issues of PMSs, there currently exists a paucity of research on the implementation of PMSs in healthcare and rehabilitation settings as well as on the factors that foster or constrain it. ${ }^{[2,5-7]}$ This study addresses this gap by identifying key determinants that will help healthcare decision makers successfully implement a PMS.

\subsection{Factors influencing PMS implementation}

A broad range of factors related to individuals, organizational context and PMS characteristics may influence the successful implementation of a PMS. It has been reported that the development of a PMS is time consuming ${ }^{[8,9]}$ and requiring specific knowledge. ${ }^{[10]}$ In healthcare settings, reported barriers to the implementation of a PMS include the availability and turnover of staff responsible for the implementation, issues related to information systems (IS), high development costs, the implementation and maintenance of such systems, and competing priorities. ${ }^{[5]}$ Factors such as a well-defined organizational vision prior to implementation, the use of feedback processes before and during implementation, ${ }^{[10,11]}$ and commitment and buy-in from decision makers were reported to be critical to the successful implementation of a PMS. ${ }^{[12-14]}$ In addition, the provision of adequate resources has been found to contribute positively to performance information use. ${ }^{[6,10,15,16]}$ Other factors such as ease of data collection as well as the accuracy, timeliness, and relevance of performance measures were also reported to be key factors for successful implementation of a PMS. ${ }^{[9,14,16]}$ Few studies have tried to appraise a large set of factors and their interactions in relation to the adoption and implementation of innovations such as the use of PMSs in healthcare organizations. ${ }^{[17]}$

\subsection{Theoretical framework}

To better understand the mechanisms by which certain factors positively or negatively influence the success of implementation, ${ }^{[17-19]}$ the Consolidated Framework for Implementation Research $(\mathrm{CFIR})^{[20]}$ was used. The CFIR is a comprehensive conceptual framework that contains five domains further divided into 33 constructs believed to have an influence on the successful implementation of an innovation. These five domains are: the external setting in which the organization evolves, the organization's own inner setting, the characteristics of the individuals involved in the implementation, the characteristics of the innovation (i.e. PMS) that is to be implemented and the process of implementation itself. The characteristics of individuals domain was not applied in our study because our unit of analysis was the organization and not the individual, leaving four domains and 28 constructs. We used the CFIR to guide the development of the interview grids and to structure data analysis.

\section{Methods}

\subsection{Study design and setting}

We conducted a qualitative longitudinal study to gain an in depth understanding of how a PMS was used in a rehabilitation hospital and what factors may explain its use. The study setting was a Canadian rehabilitation hospital that recently implemented a PMS. The setting is a typical mid-size publicly-funded urban academic rehabilitation hospital in North America providing specialized care and rehabilitation services to adults with physical disabilities. In 2010, the implementation of a PMS was identified as a main objective of the hospital's 5-year strategic plan with the goals of detecting areas of underperformance (i.e. quality improvement) and monitoring attainment of objectives and targets to be achieved. In December 2012, a first PMS was approved by the hospital board. Overall, the PMS included 30 objectives, 43 indicators, time frames and data collection dates. Table 1 provides an extract of the 2012-2013 PMS. The intended users of the PMS were healthcare executives and hospital board members.

\subsection{Data sources}

Two data sources were used: key informant (KI) interviews with rehabilitation hospital directors (e.g. CEO, along with the directors of rehabilitation services, medical affairs, rehabilitation technology, human resources and administrative services) and organizational documents. Respondents were selected for their involvement in the development and/or their role as an end-user of the PMS. Interviews with KIs were conducted at pre-implementation of the PMS (development stage) in September and October 2012 (T1) and at 10 months post-implementation in November and December 2013 (T2). In total, 14 interviews were held in each KI's office at a time convenient to each participant: seven interviews were conducted at T1 and seven at T2. In total, 9 different hospital directors were interviewed; four KIs were interviewed only once because of restructuring of the directors' board.

The interviews lasted between 60-90 minutes and were digi- 
tally recorded and transcribed. Interviews were conducted by DZ, a physiotherapist working in the organization. The interviewer was thus familiar with the study context, a characteristic which contributed to create a climate of trust between her and the participants. ${ }^{[21]}$ Two discussion guides were developed. At T1, we asked KIs open-ended questions about their conception of the PMS, its perceived advantages and intended use. We also asked about their perception of orga- nizational contextual factors such as triggers for developing and implementing a PMS, the priority of the PMS in the organization, dedicated resources and planned organizational processes related to implementation. At $\mathrm{T} 2$, the same topics were discussed to see whether or not there was a change over time with an emphasis on the implementation process and actual use of the PMS.

Table 1. Extract from the PMS for financial year 2012-2013

\begin{tabular}{|c|c|c|c|c|}
\hline Objective & $\begin{array}{l}\text { Executive } \\
\text { responsible }\end{array}$ & Indicator & Target & $\begin{array}{l}\text { Level of achievement } \\
\text { on date of revision* }\end{array}$ \\
\hline $\begin{array}{l}\text { To meet new rehabilitation needs } \\
\text { aligned with the organization's mission }\end{array}$ & $\begin{array}{l}\text { Rehabilitation } \\
\text { services executive }\end{array}$ & $\begin{array}{l}\text { Number of users served by } \\
\text { ambulatory intensive rehabilitation }\end{array}$ & $\begin{array}{l}\geq 328 \\
\text { patients }\end{array}$ & Green \\
\hline To increase organization's notoriety & CEO & $\begin{array}{l}\text { Number of applications submitted } \\
\text { for external recognition awards }\end{array}$ & 4 & Yellow \\
\hline $\begin{array}{l}\text { To achieve targets set out in the } \\
\text { 2012-2013 Management Agreement } \\
\text { between the organization and the } \\
\text { Ministry of Health }\end{array}$ & $\begin{array}{l}\text { Rehabilitation } \\
\text { services executive }\end{array}$ & $\begin{array}{l}\text { Percentage of deadlines met for } \\
\text { high priority service requests }\end{array}$ & $\begin{array}{l}90 \% \text { of patients } \\
\text { classified as } \\
\text { high priority }\end{array}$ & Green \\
\hline
\end{tabular}

* Green: target achieved between $91 \%-100 \%$ on date due; Yellow: target partially achieved (between 50\%-89\%) on date due; Red: target not reached on date due $(<50 \%)$

A total of 111 official documents (3 annual reports, 1 strategic plan, 27 minutes from hospital board meetings and 76 minutes from executive committee meetings, 2 other organizational documents and 2 PMSs reports) produced between January 2011 and April 2014 were reviewed to provide organizational and contextual information to retrospectively reconstruct events prior to PMS implementation and complement data obtained from interviews.

This study addressed the following main questions: (1) How was the PMS used, and (2) What factors enabled or constrained use of the PMS?

\subsection{Data analysis}

Success of PMS implementation was determined according to the gap between the intended versus the reported use. Intended use refers to the planned use outlined in the data sources at $\mathrm{T} 1$, while reported use refers to the actual use revealed by interviews at $\mathrm{T} 2$ or extracted from organizational documents. Moynihan's ${ }^{[22]}$ classification was used to categorize intended and reported PMS use in 3 categories: purposeful, passive and political. Purposeful use is the use of data to improve the organization's performance by more informed decisions and better allocation of resources. Passive use consists of the production and dissemination of performance information without really acting on the basis of this information. Political use is the use of performance information for advocacy purposes.

A deductive content analysis was conducted to explore fac-

Published by Sciedu Press tors influencing the use of the PMS. Such an approach is appropriate when the structure of analysis is operationalized on an existing theory or model. ${ }^{[23,24]}$ Our structured analysis was based on a predefined coding scheme based on the domains and relevant constructs from the CFIR. Data were reviewed and coded according to these pre-defined categories; however we remained open to new emergent codes throughout the analysis. Data analysis comprised several steps. Firstly, each interview transcript was verified for accuracy, read line by line and coded according to the pre-defined categories. When new codes emerged, the coding frame was changed and the transcripts were re-read according to the new structure. ${ }^{[25]}$ Transcripts were then imported into qualitative analysis software (QDA Miner 3.2.3) to ease further analysis steps. This initial coding was done by the primary author who has experience in qualitative research. Secondly, each construct was rated as a facilitator, barrier or neutral at T1 and T2, according to respondents' statements. Criteria were developed to further categorize facilitators and barriers according to importance of its contribution as major or minor, based on the number of respondents that described the construct as a facilitator or barrier (see Table 2 for criteria used to rate constructs). As a third step, we analyzed the evolution of each construct between $\mathrm{T} 1$ and T2. In addition, an explanatory matrix ${ }^{[26]}$ was produced to appraise whether each construct contributed as a facilitator or barrier to the PMS implementation using the same criteria described above.

All documents were read and coded according to the CFIR 
when relevant. Documents were also used to construct a timeline on the process of developing and implementing a PMS and to identify frequency and type of use of the PMS by directors and the hospital board.

\subsection{Methods used to assure trustworthiness of results}

Several methods were used to increase the trustworthiness of our results. To ensure confirmability, data collection and analysis was guided by theory. Moreover, the authors met regularly throughout the analysis phase to discuss progress and findings. Disagreements were discussed until a consen- sus was reached. To increase credibility, the two data sources were triangulated. The results were also verbally presented to KIs to discuss data interpretation. Respondents agreed that our interpretation corresponded to what they meant to say. Furthermore, to ensure dependability, software was used to code transcripts and to document coding decisions whereas a codebook with code definitions was produced and used when analyzing transcripts.

The research protocol was approved by the ethics review board of the Centre for Interdisciplinary Research in Rehabilitation.

Table 2. Criteria used to rate constructs according to their evolution between the two assessments

\begin{tabular}{ll}
\hline Classification & Criteria \\
\hline \multirow{3}{*}{ Major facilitator $(++)$} & $\begin{array}{l}\text { The construct has a positive influence on implementation efforts or the use of the PMS if the majority of } \\
\text { KIs }(\geq 4) \text { at T1 and T2 described the construct as a facilitator and explained or gave specific examples of } \\
\text { the positive contribution of the construct. } \\
\text { The construct has a positive influence on implementation efforts or the use of the PMS if a minority of KIs } \\
(<4) \text { at T1 and T2 described the construct as a facilitator and explained or gave specific examples of the } \\
\text { positive contribution of the construct. } \\
\text { A construct that has no influence on implementation efforts at either t1 or t2 because: (1) it was only } \\
\text { described by respondents without mentioning its influence; }(2) \text { its influence is perceived differently by } \\
\text { respondents. } \\
\text { The construct has a negative influence on implementation efforts or the use of the PMS if the majority of } \\
\text { KIs }(\geq 4) \text { at T1 and T2 described the construct as a barrier and explained or gave specific examples of the } \\
\text { negative contribution of the construct. } \\
\text { The construct has a negative influence on implementation efforts or the use of the PMS if a minority of KIs } \\
(<4) \text { at T1 and T2 described the construct as a barrier and explained or gave specific examples of the } \\
\text { negative contribution of the construct. }\end{array}$ \\
\end{tabular}

Table 3. Intended and reported use of the PMS

\begin{tabular}{|c|c|}
\hline Intended organizational use of the PMS & Reported organizational use of the PMS \\
\hline $\begin{array}{l}\text { Purposeful use } \\
\text { - Decision-making support tool by allowing the rapid } \\
\text { detection of deviations for clinical and management } \\
\text { processes improvement } \\
\text { - } \quad \text { Monitoring organizational objectives to detect } \\
\text { deviations and develop action plans to address them }\end{array}$ & $\begin{array}{l}\text { Purposeful use } \\
\text { - Tool for decision support: used infrequently as a tool for decision } \\
\text { making for improvement } \\
\text { - Strategic use to delay the achievement of a target depending on } \\
\text { other priorities or according to the external environment }\end{array}$ \\
\hline $\begin{array}{l}\text { Passive use } \\
\text { - Not mentioned }\end{array}$ & $\begin{array}{l}\text { Passive use } \\
\text { - Information tool for monitoring the level of achievement of } \\
\text { organizational objectives }\end{array}$ \\
\hline $\begin{array}{l}\text { Political use } \\
\text { - Accountability to hospital board } \\
\text { - Demonstrate the value of the organization to healthcare } \\
\text { - } \text { authorities and position it as a leader in rehabilitation } \\
\text { Negotiate organizational issues with healthcare } \\
\text { authorities }\end{array}$ & $\begin{array}{l}\text { Political use } \\
\text { - Strategic information dissemination to hospital board and to } \\
\text { healthcare authorities for accountability purposes, to promote } \\
\text { organizational performance, to enhance the organization’s } \\
\text { credibility and reputation } \\
\text { - Not used to negotiate organizational issues }\end{array}$ \\
\hline
\end{tabular}




\section{RESUlts}

\subsection{Use of the PMS}

The main results pertaining to PMS use (see Table 3) are the following: (1) PMS use was quite different from the intended one, and (2) PMS use was predominantly passive and political rather than purposeful. Important discrepancies between reported use, at $\mathrm{T} 2$, and intended use, at $\mathrm{T} 1$, were indeed identified. At T1, the primary intended use of the PMS was purposeful, as a decision-making tool to support quality improvement through timely access to objective data.
There was also strong intention to use the PMS as a political tool for accountability and negotiating power with the hospital board and local health authorities. However, at T2, the primary reported use of the PMS was passive, mainly to monitor achievement of organizational objectives rather than to support decision making for improvement as intended. Similarly, the political use was mainly limited to accountability purposes through the strategic dissemination of information from the PMS to the hospital board (around 4 times a year) and in the mandatory annual report to health authorities. The PMS was not used as a negotiating tool.

Table 4. Classification of factors as barriers or facilitators to PMS use at each assessment time

\begin{tabular}{|c|c|c|}
\hline Domains and constructs & Appraisal at T1 & Appraisal at T2 \\
\hline \multicolumn{3}{|l|}{ Outer setting } \\
\hline - Patient needs and resources & not assessed ${ }^{€}$ & not assessed ${ }^{€}$ \\
\hline • Cosmopolitanism & neutral $^{£}$ & neutral $^{£}$ \\
\hline - Peer pressure & neutral $^{£}$ & neutral $^{£}$ \\
\hline - External policy and incentives & neutral $^{£}$ & neutral $^{£}$ \\
\hline \multicolumn{3}{|l|}{ Innovation characteristics } \\
\hline - Innovation source & facilitator ++ & facilitator ++ \\
\hline - Evidence strength and quality & facilitator ++ & barrier ++ \\
\hline - Relative advantage & facilitator ++ & facilitator ++ \\
\hline - Adaptability & facilitator ++ & barrier + \\
\hline - Trialability & not applicable ${ }^{\S}$ & not applicable ${ }^{\S}$ \\
\hline - Complexity (reverse rated) & facilitator ++ & facilitator ++ \\
\hline - Design quality and packaging & facilitator ++ & facilitator ++ \\
\hline - Cost & not applicable ${ }^{\varphi}$ & not applicable ${ }^{\varphi}$ \\
\hline \multicolumn{3}{|l|}{ Inner setting } \\
\hline - Structural characteristics & not assessed ${ }^{\xi}$ & not assessed ${ }^{\xi}$ \\
\hline - Networks and communications & barrier + & facilitator ++ \\
\hline - Culture & barrier + & facilitator + \\
\hline \multicolumn{3}{|l|}{ - Implementation Climate } \\
\hline - Tension for change & facilitator ++ & facilitator ++ \\
\hline - Compatibility & facilitator ++ & barrier + \\
\hline - Relative priority & facilitator ++ & barrier + \\
\hline - Organizational incentives and rewards & not applicable & not applicable \\
\hline - Goals and feedback & facilitator ++ & barrier + \\
\hline - Learning climate & barrier ++ & facilitator + \\
\hline \multicolumn{3}{|l|}{ - Readiness for Implementation } \\
\hline - Leadership commitment & facilitator ++ & facilitator ++ \\
\hline - Available resources & barrier ++ & barrier++ \\
\hline - Access to knowledge and information & facilitator ++ & facilitator ++ \\
\hline \multicolumn{3}{|l|}{ Process } \\
\hline - Planning & barrier ++ & barrier ++ \\
\hline - Engaging & facilitator ++ & facilitator ++ \\
\hline - Executing & facilitator + & facilitator + \\
\hline - Reflecting and evaluating & barrier ++ & barrier ++ \\
\hline
\end{tabular}


Table 5. Summary of facilitators to PMS implementation and use

\begin{tabular}{l}
\hline Innovation Characteristics \\
\hline Innovation source: development and implementation of PMS was an internally \\
initiated project to address an organizational need to change.
\end{tabular}

Relative Advantage: Several advantages of PMS were reported: (1) PMS implementation promoted the development of a common vision among directors and encouraged collaboration; (2) PMS information supported decisions based on objective data rather than intuition and (3) PMS was useful for monitoring organizational performance and strategic orientations and quickly detecting gaps. Complexity: PMS was perceived to be simple and easy to use.

Design Quality and Packaging: directors appreciated the simple, short, user-friendly and color-coded visual presentation of the PMS information that allowed for quick retrieval of the information and identification of the degree of achievement of each indicator.

Inner setting
Network and communication: Directors reported that good team relations led to a
climate of trust and spawned more sharing and faster disseminating of
performance information through formal communication channels (e.g., planned
meetings) as well as through informal communications channels (e.g., phone
calls).
Culture: PMS implementation contributed to foster the development of a
measurement culture.
Tension for change: The PMS was perceived by directors as addressing the need
for objective information to monitor attainment of organizational goals, and to
detect opportunities for quality improvement.

Learning climate: A favourable learning climate was developed with the CEO's leadership and ability to rally all executives and ensure a collaborative environment.

Leadership commitment: Continuous leadership commitment of all PMS intended users, particularly the CEO's leadership, were key factors to PMS implementation.

Access to knowledge and information: Directors reported having the necessary knowledge from previous education and/or work experience about measurement concepts and development of a PMS.

Process
Engaging: The CEO, executives and hospital board members were consistently
engaged throughout the implementation project.

Executing: Directors reported providing data for indicators in a timely manner.

\section{Respondents quotes}

"The project was initiated by the CEO, but it was also something that was discussed in various consultations with decision makers and during the development of the strategic plan.” KI 1

"The added value of such a tool would be that we could rely on objective data when we are making decisions.” KI 5

"I feel that this is a very simple tool.” KI 7

"I like the presentation; we have all the information at a glance." KI 2

\section{Respondents quotes}

"We are no longer in power struggle, either way I do not feel we are.” KI 3

“Our measurement culture is not yet developed enough.” KI 7

"You know, given that every penny that you get must be used very judiciously and sparingly, I think [the PMS] is a necessary tool for making good decisions, for getting a good picture of the organization.” KI 2

"Recently, we assessed our satisfaction with the functioning of the executive committee and it was very positive. Everybody appreciates the climate”. KI 1

“This project was initiated following the CEO's vision. We knew it would be materialized because it was endorsed by the CEO.” KI 7

"I think we have the expertise to develop a performance measurement system.” KI 5

\section{Respondents quotes}

"The CEO was actively involved in the entire PMS development process, however all the directors were also consulted, it was a participatory approach.” KI 8

"Each of us is responsible for specific indicators; we provide indicators data to the project leader as required” KI 2

\subsection{Factors that facilitated or constrained PMS use}

The analysis revealed that the implementation of a PMS is a multi-factorial and dynamic process. Indeed, from a total of 28 CFIR constructs, 20 were identified as factors influencing PMS implementation and use. More specifically, $60 \%$ of the factors were categorized as facilitators whereas $40 \%$ as barriers. However, $40 \%$ of the factors evolved over time: for example, a factor identified as a facilitator at $\mathrm{T} 1$ could have evolved to a barrier at $\mathrm{T} 2$ or vice versa. Table 4 provides a classification of factors as barriers or facilitators to PMS use at each assessment time.
In the following section, barriers and facilitators will be presented following the structure of the CFIR framework (outer setting; innovation characteristics; inner setting and process). Tables 5 and 6 provide a summary of barriers and facilitators of PMS implementation and use, along with corresponding respondents' quotes.

\subsubsection{Outer setting}

Not one factor related to the outer setting (cosmopolitanism, peer pressure and external policy and incentives) influenced the implementation of the PMS. Nevertheless, respondents reported a lack of external policy and incentives for the de- 
velopment and implementation of PMS at the organizational level. Respondents felt that although the political discourse on performance is omnipresent in the Quebec health care system, rehabilitation organizations have no real pressure, other than their internal needs, for PMS implementation.

\subsubsection{Innovation characteristics}

Four factors (innovation source, relative advantage, lack of complexity and design quality and packaging) were identified as major facilitators at both T1 and T2 to PMS implementation and use. Internal initiation of PMS development was identified as a major facilitator because it addressed an important organizational need for change. Respondents also perceived several advantages to PMS implementation, such as its usefulness for monitoring organizational performance. Finally, PMS was perceived to be user-friendly with a visual design that allowed for quick analysis of information.
Two factors (evidence strength/quality, and adaptability) evolved from facilitators to barriers. Concerning evidence strength/quality of PMS, respondents felt that the PMS should integrate a restricted number of indicators reflecting the various activities of hospital departments. Those indicators should be based on prioritized annual objectives with a balance between strategic and operational indicators. At T2, the majority of respondents were disappointed by the absence of clinical indicators and the fact that the selected indicators did not allow for benchmarking. Overall, the evidence strength/quality of the PMS did not reach the expected levels and partially met the needs of hospital directors. Furthermore, while KIs expressed the need for the PMS to be adaptable, the addition or removal of indicators to adjust to the changing organizational environment or to meet new information needs was not possible.

Table 6. Summary of barriers to PMS implementation and use

\begin{tabular}{l}
\hline Innovation Characteristics \\
Evidence strength and quality: Directors were disappointed by the quality \\
of the PMS for three main reasons: (1) absence of balance between \\
strategic and operational indicators; (2) absence of clinical indicators and \\
(3) selected indicators did not allow for benchmarking. \\
Adaptability: Directors reported the lack of flexibility of the PMS: the \\
addition or removal of indicators to adjust to the changing organizational \\
environment or to meet new information needs was not possible. \\
Inner setting \\
Compatibility: Directors perceived that the PMS only partially met their \\
needs: the PMS allowed for monitoring of organizational goals and \\
strategic planning but was not useful for decision making. \\
Goals and feedback: Directors reported a significant gap between the \\
intended frequency of feedback about PMS information (once a month) \\
and the actual one (five times). \\
Relative Priority: Directors shared the perception that the priority of the \\
PMS declined over time.
\end{tabular}

Available resources: Directors perceived that resources available for PMS development and implementation were insufficient (e.g. time, development of information systems).

\section{Respondents quotes \\ "The clinical component is absent from the dashboard; it is not there but it is our core business.” KI 3}

"What worries me is that the dashboard has not changed much since last year; there are two versions and both are very similar.” KI 7

\section{Respondents quotes}

"The dashboard does not meet our needs; we cannot do benchmarking with this tool” KI 6

"We need to have regular feedback on performance information to motivate ourselves to use it.” KI 1

"I think we have just been too busy doing other things, more specifically all the reorganization following the budgetary constraints.” KI 6

"You know, this project was developed along with all the other organizational projects during our executive committee meetings. It was not possible for us to have any additional meetings beyond that to work on this project.” KI 8

\section{Respondents quotes}

"In hindsight, in order to have done this project right, we should have had a plan” KI 4

"We did not do a global review of the entire project. I think we should do one and clarify what are our expectations with this dashboard.” KI 7 sharing of individual reflections and experiences with PMS use.

\subsubsection{Inner setting}

Three factors (leadership commitment, tension for change and access to knowledge) remained major facilitators throughout implementation of the PMS. Leadership com-

Published by Sciedu Press mitment of the CEO for developing and implementing the PMS was continuous throughout the project and thus was a major facilitator. Indeed during both evaluation times, KIs stated that the CEO was able to rally the hospital board and 
the directors to follow through with this project. Furthermore, he enhanced and strengthened the perceived value and the importance of implementing a PMS in the organization. Thus the tension or need for a PMS, already present at T1, remained a strong facilitator throughout its implementation. Furthermore, implementation of PMS was also facilitated through easy access to knowledge: at both evaluation times, KIs felt confident about their level of knowledge on measurement concepts and development of a PMS, given their education and/or work experience.

Three factors evolved from barriers to facilitators (culture, learning climate and networks and communication) as the PMS implementation progressed. At T1, the PMS implementation was seen as a means to improve a sub-optimal measurement culture. At T2, although several KIs stated that the PMS improved the measurement culture in the organization, they recognized that the strong leadership of the CEO and the importance emphasized towards objective information were key contributing factors.

CEO leadership has also exerted a decisive influence on several other organizational factors. Indeed, at T1 KIs reported that the learning climate was not favourable to PMS implementation because power between directors was unequally distributed and that work was often done in silos with little information sharing. At T2, a deliberate restructuring of the executive committee by the CEO significantly improved the learning climate. This created a climate of trust where collaborative teamwork and information sharing was strongly encouraged. The improvement of work relations has led to more sharing and a more rapid dissemination of information via informal communications (calls telephone, email, etc.) Furthermore, regular meetings played a major facilitating role at $\mathrm{T} 1$ and $\mathrm{T} 2$ as they provided a formal structure to share information on the development of the PMS and to discuss PMS results. Thus, a more positive learning environment and an improved quality of communications between the directors facilitated PMS implementation.

Four factors remained or evolved as barriers: relative priority, available resources, compatibility and goals and feedback. The relative priority of PMS implementation to other organizational projects evolved from a major facilitator at $\mathrm{T} 1$ to a minor barrier at T2. At T1, merging of the organization required major administrative restructuring of top management. In spite of this context, the board of directors decided to keep the PMS as an organizational goal to be accomplished during 2012-2013. However at T2, KIs had a shared perception that the PMS was developed and implemented with limited organizational resources and therefore, the project was not granted the desired priority. Indeed, the lack of available re- sources remained a major barrier through the whole project. There was no protected time for the development and implementation of the PMS aside from the pre scheduled meetings of the steering committee that covered several topics including the PMS project. However at T2, appointment of a formal implementation leader responsible for the management of the PMS project was identified by all KIs as an important facilitator to PMS implementation. Given the lack of additional resources to develop an IS, administrative and clinical information was collected in several IS that were not compatible and required manual manipulation of data. Furthermore, limited resources have also resulted in the inability to invest a lot of time in research and development of indicators aligned with directors' information needs. Thus at T2, KIs perceived that the PMS was only partially compatible with their needs. Indeed, KIs reported that the PMS allowed monitoring of organizational goals and strategic planning but was not very useful for decision making because it had limited benchmarking capacity and lacked operational indicators reflecting their main activities or concerns. Finally, feedback on performance information evolved as a minor barrier because of the significant gap between the intended frequency of feedback and the actual one. While the monitoring of indicators, targets and organizational goals was intended to be on the directors' board agenda on a monthly basis, the PMS was only discussed five times in FY 2013-2014.

\subsubsection{Process}

The Process domain contains four constructs (planning, engaging, executing and reflecting and evaluating) that were difficult to disentangle from each other. Planning remained a major barrier throughout the project. While KIs reported that a global plan for PMS development was established by the CEO, they felt that some activities (e.g. common vision of PMS goals, resources required) were missing from the planning phase and not addressed during implementation. Engaging appropriate individuals through PMS development and implementation was a major facilitator throughout the study. Indeed, the involvement of directors in the execution of implementation activities such as providing the necessary on-time indicators information required for the PMS contributed to their engagement to this project. Reflecting and evaluating was a major barrier throughout the process as KIs criticized the absence of dedicated time for team debriefing during directors' board meetings at both T1 and T2. At T2, respondents expressed the need to appraise their satisfaction, their use and potential improvements to the PMS.

\section{Discussion}

To our knowledge, this is the first rehabilitation-based study examining PMS use among healthcare decision makers. Our 
results showed that success of implementation was moderate, as the reported use of the PMS by decision makers was less than anticipated. More specifically, reported purposeful and political PMS use was less than intended and passive use prevailed. Indeed, decision makers primarily used the PMS to monitor level of achievement of organizational objectives (passive use) and to disseminate PMS information to the hospital board and health authorities for accountability purposes. PMS was less often used to promote the organization's performance and enhance its credibility and reputation (political use).

Our results also showed that PMS implementation and use was a complex and dynamic process influenced by a variety of evolving and interrelated factors. Among the 28 CFIRrelated constructs assessed, 20 were identified as factors influencing PMS use. The number of factors categorized as facilitators exceeded those categorized as barriers. Indeed, $60 \%(12 / 20)$ of factors remained or evolved as facilitators through the development and the implementation of a PMS in this rehabilitation setting and $40 \%(8 / 20)$ remained or evolved as barriers. While continuous leadership engagement was identified as a major facilitator for PMS implementation, major barriers such as a lack of planning, sub-optimal PMS quality and insufficient resources seemed to have dampened its use. Those four factors will be discussed below, given their interrelations and their predominant influence on PMS implementation and use.

In our study, commitment and support of top management for the PMS was a key facilitator and prerequisite for PMS adoption, development and implementation. Particularly, the CEO's strong leadership was essential to initiating the project, communicating the value of PMS implementation for the organization, rallying executives and hospital board members around a common vision, and enhancing their commitment to the PMS project despite many other competing priorities. This finding is consistent with numerous studies in which high commitment and constancy of commitment of senior management has been identified as a key success factor in PMS implementation. ${ }^{[15,27-30]}$

The leader should also play an important role in promoting the development of an implementation plan, an essential step that has been documented as one of the determinants of successful implementation and use of PMSs. ${ }^{[5,15,31-34]}$ However, in the present study, the planning stage seemed to be insufficient and therefore negatively influenced PMS use.

Indeed, insufficient planning directly and indirectly affected the relevance of the PMS developed and, ultimately, its use. As a first step during the planning stage, the intended users and their needs should have been identified and the goals of the PMS should have been made explicit. However, such planning activities seemed to have been omitted, according to KI's interviews, and as a result, the PMS was partially compatible with the information needs of executives. Second, the lack of planning seemed to have contributed to insufficient allocation of resources for PMS development. The importance of adequate resources on success of PMS implementation has been extensively documented. ${ }^{[5,15,28,35-37]}$ Scarce resources resulted in the inability to invest much time in the search and development of indicators aligned with the information needs of directors. Moreover, no additional resources were available to develop an IS to support PMS implementation. PMS development and implementation had to be done alongside other tasks of the directors and using formal management structures such as the directors' board meetings. As a result, KIs reported that the indicators were too global, undiversified, sometimes outdated, and not operationalized enough for decision making. Relevancy and accuracy of indicators, as well as timeliness and frequency of feedback were reported to be important factors influencing PMS use in other studies. ${ }^{[5,15,34,38]}$

In our study, PMS was used predominantly for accountability purposes and to a much lesser extent for quality improvement. This may suggest that pursuing both the objective of improving accountability and improving decision making may be difficult to achieve in the same PMS. ${ }^{[39,40]}$ In a perspective of political use where accountability is strong, fear of failure can reduce risk-taking by organizations that may choose less challenging targets to ensure achievement. One way to address this problem is to dissociate performance measurement for internal improvement purposes from accountability purposes early on in the development process. Indeed, organizations can develop distinct but interrelated PMSs to reconcile those different purposes and address the different information needs of decision makers. ${ }^{[39-42]}$ This would allow the inclusion of a more strategic and "accountability" oriented component for hospital boards or other external users, and a more operational and "decision" oriented component for executive committees or other internal users. The development of a more operational component of a PMS may encourage organizations to develop PMSs with characteristics (e.g., quality, relevance, usefulness of indicators) that will adequately meet the needs of decision makers.

In light of these results, the following four key recommendations are proposed that may enhance PMS implementation and use:

(1) To involve PMS users in the selection of objectives, indicators and targets as soon as possible (i.e. during the planning phase). ${ }^{[15,43]}$ During the planning phase, 
it is also important to be explicit about the relevance of each indicator, its use and who will be accountable for it. ${ }^{[44]}$

(2) To determine the available resources early in the PMS development and implementation process, in order to align resources with the scope of the intended PMS and with users' information needs. This will help avoid disappointments or gaps between a desired PMS and what is possible and realistic for the organization to develop. ${ }^{[15,33]}$

(3) To ensure that the final PMS is aligned with the in formation needs of intended users. This will avoid content that is focused on overall performance, and will favour content with specific information that is actionable and appropriate within each user's level of responsibilities over the achievement of any given objective. ${ }^{[15,38,39]}$

(4) To develop distinctive PMS sections with accountability indicators, as well as quality targets, if accountability purposes appear to override internal quality improvement. ${ }^{[39-42]}$

\section{Study limitations}

Most of the findings are based on the perceptions of respondents at two different times. In addition to a recall bias, it is also possible that a social desirability bias was present and participants, because of their position as directors, overstated their use of the PMS. However, our results indicate that participants reported as well their positive experiences as their negative experiences and dissatisfactions related to the development and the implementation of the PMS. Second, qualitative research involves unavoidable elements of researchers' subjectivity. For example, in our study we have decided that when a majority of participants reported positive/negative influence of a factor on PMS implementation, this factor was qualified as having major influence vs. minor influence. However, several methods were put in place to minimize biases in interpreting results, such as a review of all findings by the authors and a validation by the respondents of the accuracy of our interpretations of PMS implementation and use. Third, we conducted study in a typical North
American rehabilitation hospital in a public-funded health system. This environment should be taken into account when transferring our results to other types of organizations or health systems' governance. While generalization may be limited, the transferability of our findings is reinforced because of the rich and comprehensive description of the case, the context and the factors influencing PMS implementation that allows readers to extract the information relevant to their setting. ${ }^{[45,46]}$ An analytical generalization ${ }^{[45]}$ might be made concerning the factors found to influence PMS implementation and use. These factors are derived from a theoretical framework (CFIR) and are corroborated by empirical research on PMS implementation in different types of public organizations including healthcare organizations. Nonetheless, this study should be replicated in other rehabilitation settings to establish its generalizability with more confidence.

\section{Conclusions}

This study examined the influence of a set of comprehensive theory-driven factors on PMS use for the first time in a rehabilitation setting. It revealed that PMS was used more frequently for monitoring and accountability and less for decision making or improvement, as intended. This predominant use was explained by insufficient planning about users' needs, intended use and content of the PMS combined with insufficient resources and other competing organizational priorities. This resulted in a sub-optimal PMS that was poorly compatible with information needs of directors. This study shows that PMS implementation and use is a complex and dynamic process influenced by multiple evolving and interrelated factors. Furthermore, our results confirm that the positive or negative influence of each factor is context dependent, thus highlighting the relevance of conducting additional studies in various healthcare settings. This will further help determine and understand the key factors influencing PMS implementation and use, along with the relative importance of each factor and their interactions.

\section{CONFlicts of InTEREST Disclosure}

The authors declare they have no conflicts of interest.

\section{REFERENCES}

[1] McDavid J, Huse I, Hawthorn L. Program Evaluation and Performance Measurement: An Introduction to Practice. Thousand Oaks, CA: SAGE Publications; 2013. 560 p.

[2] Wardhani V, Utarini A, Pieter van Dijk J, et al. Determinants of quality management systems implementation in hospitals. Health Policy. 2009; 89(3): 239-51. PMid: 18752866. http://dx.doi.org/10. 1016/j.healthpol.2008.06.008

[3] Kanji G, Moura e Sá P. Sustaining healthcare excellence through performance measurement. Total Quality Management \& Business Excellence. 2003; 14(3): 269-89. http://dx.doi.org/10.1080 /1478336032000046607

[4] Smith PC, Mossialos E, Papanicolas I, et al. Part I - Principles of performance measurement. In: Performance Measurement for Health 
System Improvement: Experiences, Challenges and Prospects [Internet]. New York, NY: Cambridge University Press; 2010. http: //dx.doi.org/10.1017/CB09780511711800

[5] Adair CE, Simpson L, Birdsell JM, et al. Performance Measurement Systems in Health and Mental Health Services: Models, Practices and Effectiveness. A State of the Science Review. The Alberta Heritage Foundation for Medical Research. 2003.

[6] Kaplan HC, Brady PW, Dritz MC, et al. The Influence of Context on Quality Improvement Success in Health Care: A Systematic Review of the Literature. The Milbank Quarterly. 2010; 88(4): 500-59. PMid: 21166868. http://dx.doi.org/10.1111/j.1468-0009. 2010.00611.x

[7] Elg M, Kollberg B, Palmberg K. Performance measurement to drive improvements in healthcare practice. International Journal of Operations \& Production Management. 2013; 33(11/12): 1623-51. http://dx.doi.org/10.1108/IJOPM-07-2010-0208

[8] Goddard M, Mannion R, Smith P. Performance indicators. All quiet on the front line. Healt Serv J. 1998; 108: 24-6. PMid: 10180005.

[9] Chan YCL, Ho SJK. The use of balanced scorecard in Canadian hospitals. Advances in Management Accounting. 1999.

[10] Aguilera J, Walker K. A new framework to ensure excellence in patient-focused care: the nursing directorate's Balanced Scorecard approach. Asia Pacific Journal of Health Management. 2008; 3(2): 25-9.

[11] Inamdar N, Kaplan R, Bower M, et al. Applying the balanced scorecard in healthcare provider organizations. Journal of Healthcare Management. 2002; 47(3): 179-96. PMid: 12055900.

[12] Curtright J, Stolp-Smith S, Edell E. Strategic performance management: Development of a performance measurement system at the Mayo Clinic. Journal of Healthcare Management. 2000; 45(1): 58-68. PMid: 11066953.

[13] Smith A, Mainland J, Blais I. Managing strategy to enhance care for children. Healthcare Quarterly. 2011; 14: 21-6. PMid: 22008569. http://dx.doi.org/10.12927/hcq. 2011.22535

[14] Dyball M, Cummings L, Yu H. Adoption of the concept of a Balanced Scorecard within NSW Health: An exploration of staff attitudes. Financial Accountability \& Management. 2011; 27(3): 335-61. http://dx.doi.org/10.1111/j.1468-0408.2011.00528.x

[15] Lemire M, Demers-Payette O, Jefferson-Falardeau J. Dissemination of performance information and continuous improvement : a narrative systematic review. Journal of Health Organization and Management. 2013; 27(4): 449-78. PMid: 24003632. http://dx.doi . org/10.1108/JHOM-08-2011-0082

[16] Chang WC, Tung YC, Huang CH, et al. Performance improvement after implementing the Balanced Scorecard: A large hospital's experience in Taiwan. Total Quality Management \& Business Excellence. 2008; 19(11): 1143-54. http://dx.doi.org/10.1080/1478336 0802323560

[17] Greenhalgh T, Robert G, Macfarlane F, et al. Diffusion of Innovations in Service Organizations: Systematic Review and Recommendations. Milbank Quarterly. 2004; 82(4): 581-629. PMid: 15595944. http://dx.doi.org/10.1111/j.0887-378X.2004.00325.x

[18] Simpson DD, Flynn PM. Moving Innovations into Treatment: A Stage-based Approach to Program Change. J Subst Abuse Treat. 2007; 33(2): 111-20. PMid: 17434704. http://dx.doi.org/10. $1016 / j \cdot j$ sat. 2006.12 .023

[19] Fixsen DL, Naoom SF, Blase KA, et al. Implementation research: a synthesis of the literature. Tampa Florida University of South Florida Louis De La Parte Florida Mental Health Institute National Implementation Research Network. 2005.

[20] Damschroder L, Aron DC, Keith RE, et al. Fostering implementation of health services research findings into practice: a consolidated framework for advancing implementation science. Implementation Science. 2009; 4(50): 1-15. http://dx.doi.org/10.1186/174 8-5908-4-50

[21] Stake RE. Case studies. Handbook of Qualitative Research. New York: Sage Publications; 1994. 435-54 p.

[22] Moynihan DP. Through A Glass, Darkly: Understanding the Effects of Performance Regimes Public Performance and Management Review. 2009; 32(4): 592-603. http://dx.doi.org/10.2753/PMR 1530-9576320409

[23] Hsieh H-F, Shannon S. Three Approaches to Qualitative Content Analysis. Qualitative Health Research. 2005; 15(9): 1277-88. PMid: 16204405. http://dx.doi.org/10.1177/1049732305276687

[24] Elo S, Kyngas $\mathrm{H}$. The qualitative content analysis process. Journal of Advanced Nursing. 2008; 62(1): 107-15. PMid: 18352969. http://dx.doi.org/10.1111/j.1365-2648.2007.04569.x

[25] Blais M, Martineau S. L'analyse inductive générale: description d'une démarche visant à donner un sens à des données brutes [A general inductive analysis: description of an approach to make sense of the raw data]. Recherches qualitatives. 2006; 26(2): 1-18.

[26] Miles MB, Huberman M. Qualitative Data Analysis: An Expanded Sourcebook. Beverly Hills, CA: Sage Publications; 1994.

[27] Weiner BJ, Shortell SM, Alexander J. Promoting clinical involvement in hospital quality improvement efforts: the effects of top management, board, and physician leadership. Health Serv Res. 1997; 32(4): 491-510. PMid: 9327815.

[28] Radnor ZJ, Lovell B. Success factors for implementation of the balanced scorecard in a NHS multi-agency setting. International Journal of Health Care Quality Assurance. 2003; 16(2): 99-108. http://dx.doi.org/10.1108/09536860310465618

[29] Kollberg B. Exploring the use of Balanced Scorecards in a Swedish health care organization. Linköpings, Sweden: Linköpings universitet; 2003.

[30] Bourne M, Mills J, Wilcox M, et al. Designing, implementing and updating performance measurement systems. International Journal of Operations \& Production Management. 2000; 20(7): 754-71. http://dx.doi.org/10.1108/01443570010330739

[31] Schalm C. Implementing a balanced scorecard as a strategic management tool in a long-term care organization. Journal of Health Services Research \& Policy. 2008; 13: 8-14. PMid: 18325162. http://dx.doi.org/10.1258/jhsrp. 2007.007013

[32] Neely A. The challenges of performance measurement. Management Decision. 2004; 42(8): 1017-23. http://dx.doi.org/10.1108 100251740410555515

[33] Kanouse DE, Spranca M, Vaiana M. Reporting about health care quality: a guide to the galaxy. Health Promotion Practice. 2004; 5(3): 222-31. PMid: 15228776 . http://dx.doi.org/10.1177/15248 39904264511

[34] De Waal AA, Counet H. Lessons learned from performance management systems implementations. International Journal of Productivity and Performance Management. 2009; 58(58): 367-90.

[35] Verzola A, Bentivegna R, Carandina G, et al. Multidimensional evaluation of performance: experimental application of the balanced scorecard in Ferrara university hospital. Cost Effectiveness and Resource Allocation. 2009; 7: 15-22. PMid: 19737409. http: //dx.doi.org/10.1186/1478-7547-7-15

[36] De Lancer Julnes P, Holzer M. Promoting the utilization of performance measures in public organizations: an empirical studyof factors affecting adoption and implementation. Pub Adm Rev. 2001; 61(6): 693-708. http://dx.doi.org/10.1111/0033-3352.00140

[37] Bourne M, Neely A, Mills J, et al. Why some performance measurement initiatives fail: lessons from the change management lit- 
erature Int J Business Performance Management. 2003; 5: 245-69. http://dx.doi.org/10.1504/IJBPM. 2003.003250

[38] Cheng SM, Thompson LJ. Cancer Care Ontario and integrated cancer programs. Journal of Health Organization and Management. 2006; 20(4): 335-43. PMid: 16921817. http://dx.doi.org/10.1108 /14777260610680131

[39] Wisniewski M, Stewart D. Performance measurement for stakeholders: the case of Scottish local authorities. International Journal of Public Sector Management. 2004; 17(3): 222-33. http: //dx.doi.org/10.1108/09513550410530153

[40] McDavid J, Huse I. Legislator Uses of Public Performance Reports: Findings From a Five-Year Study. American Journal of Evaluation. 2012; 33(1): 7-25. http://dx.doi.org/10.1177/10982140114 05311

[41] Yap C, Siu E, Baker GR, et al. A Comparison of Systemwide and Hospital-Specific Performance Measurement Tools. Journal of
Healthcare Management. 2005; 50(4): 251-62. PMid: 16130808.

[42] McAdam R, Hazlett S, Casey C. Performance management in the UK public sector: Addressing multiple stakeholder complexity. International Journal of Public Sector Management. 2005; 18(3): 256-73. http://dx.doi.org/10.1108/09513550510591542

[43] Taylor J. Strengthening the link between performance measurement and decison making Public Administration Review. 2009; 87(4): 85371. http://dx.doi.org/10.1111/j.1467-9299.2009.01788 . $\mathrm{x}$

[44] GAO. Managing for Results: Enhancing Agency Use of Performance Information for Management Decision Making. U.S. Government Printing Office. 2005.

[45] Yin R. Case study research: design and methods. London: Sage Publications; 2003.

[46] Guba E, Lincoln Y. Fourth generation evaluation. Newbury Park: Sage Publications; 1989. 